\title{
Characterization of protein arginine methyltransferase of TgPRMT5 in Toxoplasma gondii
}

\author{
Min Liu ${ }^{1}$, Fen-Xiang Li ${ }^{1}$, Chun-Yuan Li ${ }^{1}$, Xiao-Cong Li ${ }^{1}$, Long-Fei Chen ${ }^{1}$, Kun Wu${ }^{1}$, Pei-Liang Yang ${ }^{2}$, Zhi-Fa Lai ${ }^{3}$, \\ Ting-kai Liư ${ }^{4}$, William J. Sullivan Jr. ${ }^{4,5}$, Liwang Cui ${ }^{6}$ and Xiao-Guang Chen ${ }^{{ }^{*}}$
}

\begin{abstract}
Background: Protein arginine methylation is a prevalent post-translational modification. The protein arginine methyltransferase family (PRMT) is involved in many cellular processes in eukaryotes, including transcriptional regulation, epigenetic regulation, RNA metabolism, and DNA damage repair. Toxoplasma gondii, an opportunistic protozoan parasite, encodes five conserved PRMTs. PRMT5 is thought to be responsible for substantial PRMT activity in T. gondii; however, it has not yet been characterized.

Methods: We tagged the $3^{\prime}$ end of the endogenous TgPRMT5 genomic locus with sequence encoding a $3 \mathrm{X}$ hemagglutinin (HA) epitope. IFA and WB were performed to check the expression and subcellular localization of TgPRMT5 in tachyzoites and bradyzoites. In vitro methylation assays were performed to determine whether endogenous TgPRMT5 has arginine methyltransferase activity.

Results: IFA and WB results showed that T. gondii PRMT5 (TgPRMT5) was localized in the cytoplasm in the tachyzoite stage; however, it shifts largely to the nuclear compartment in the bradyzoite stage. The in vitro methylation showed that TgPRMT5 has authentic type II PRMT activity and forms monomethylarginines and symmetric dimethylarginines.

Conclusions: We determined the expression and cellular localization of TgPRMT5 in tachyzoites and bradyzoites and confirmed its type II PRMT activity. We demonstrated the major changes in expression and cellular localization of TgPRMT5 during the tachyzoite and bradyzoite stages in T. gondii. Our findings suggest that TgPRMT5 protein may be involved in tachyzoite-bradyzoite transformation.
\end{abstract}

Keywords: Histone, Chromatin, Parasites, Epigenetics, Methylation, Bradyzoites

\section{Background}

Toxoplasma gondii ( $T$. gondii) is an obligate intracellular parasite of the phylum Apicomplexa. This species is the causative agent of toxoplasmosis, which is estimated to infect $16-40 \%$ of the population of the USA and up to $80 \%$ of the population in other countries [1]. Toxoplasma gondii can differentiate from a rapidly replicating tachyzoite stage to a latent cyst form, the bradyzoite stage. Bradyzoite cysts can remain in tissues of the host

\footnotetext{
*Correspondence: xgchen2001@hotmail.com

1 Department of Pathogen Biology, Guangdong Provincial Key Laboratory of Tropical Disease Research, School of Public Health, Southern Medical University, Guangzhou 510515, Guangdong, People's Republic of China Full list of author information is available at the end of the article
}

for its lifetime without health consequences. However, when the host immunity is attenuated, bradyzoites can revert to actively replicating tachyzoites, causing lifelong chronic infection. Thus, understanding the molecular mechanisms underpinning conversion between these stages may identify novel targets for clinical treatment.

Stage-specific gene expression is controlled by the concerted actions of specific transcription factors and repressors, translational repression, and epigenetic mechanisms [2]. Significant alterations in the expressed transcriptome and remodeling of chromatin structure, a major mechanism restricting and regulating access to genomic DNA, are occurring during the conversion between the tachyzoite and bradyzoite 
stages. Toxoplasma gondii has a full complement of histone-modifying enzymes, histones and variants [3]. Characterization of the T. gondii histone-modifying enzymes will provide a better understanding of the role of epigenetic regulation in gene expression in pathogenic apicomplexan parasites like $T$. gondii.

Gene expression can be negatively or positively modulated by methylation of arginine and lysine residues in histones. Moreover, both arginine and lysine methyltransferases are proposed to play an important role of gene expression regulators in T. gondii [4].

Structurally, each protein arginine methyltransferase family (PRMT) shares a conserved methyltransferase domain with subdomains for binding to S-adenosylL-methionine (SAM), a methyl donor and substrate proteins. Five putative arginine methyltransferases have been revealed by the bioinformatic analysis of the Toxoplasma genome sequence (http://www.toxod b.org) [5], and two PRMTs have been characterized in T. gondii [4-6]. PRMT1 (TGME49_219520) is reported to mediate methylation of arginine 3 of $\mathrm{H} 4$, while PRMT4 (TgCARM1) catalyzes methylation of arginine 17 of $\mathrm{H} 3$ and has been attributed to gene activation [5]. PRMT5 is a type II methyltransferase associated with transcriptional repression in other species $[7,8]$. It is associated with multiple protein complexes and mediates diverse functions including RNA processing, transcriptional regulation, and muscle as well as germ line differentiation [9]. However, in T. gondii, the functions of PRMT5 remain poorly understood.

Here, we report the identification and characterization of the PRMT5 homolog, referred as TgPRMT5, in T. gondii. TgPRMT5 (TGGT1_215560) is located on chromosome X, spans $2940 \mathrm{bp}$, and contains no introns. It encodes a protein of 979 amino acids with a predicted molecular mass of $107 \mathrm{kDa}$. The PROSITE database (http://us.expasy.org/prosite/) was used to search for predicted protein motifs in TgPRMT5. TgPRMT5 contains a conserved SAM-dependent methyltransferase PRMT-type domain as the PROSITE database showing it ranging from 601 to 957 amino acids. TgPRMT5 is an evolutionarily conserved protein with type II PRMT activity toward histone H3 arginine-26 (H3R26) and histone $\mathrm{H} 4$ arginine-3 (H4R3). Mammalian PRMT5 localizes to both the cytoplasm and the nucleus [10]. It has been proposed that PRMT5 relocates from nucleus to cytoplasm, where it may play a role in pluripotency regulation $[11,12]$. TgPRMT5 is expressed in both tachyzoite and bradyzoite stages, and it is differentially localized between these stages, suggesting that TgPRMT5 might play a role in tachyzoite-bradyzoite conversion.

\section{Methods}

\section{Parasite culture and nucleic extraction}

The avirulent Pru $\Delta k u 80$ (Prugniaud) strain of T. gondii was maintained by serial passage in human foreskin fibroblasts (HFF) cultivated in Dulbecco's modified Eagle medium supplemented with $1 \%(\mathrm{v} / \mathrm{v})$ heat-inactivated fetal bovine serum and $25 \mu \mathrm{g} / \mathrm{l}$ gentamicin antibiotic (Life Technologies). To induce bradyzoite formation, approximately 50,000 tachyzoites were inoculated onto confluent HFF monolayers in T25 flasks with culture medium. Two to three hours post-infection, the culture medium was replaced with a $\mathrm{pH} 8.2$ medium, which was replaced daily. Parasite DNA was isolated from the parasite pellet by proteinase $\mathrm{K}$ digestion and phenol/chloroform extraction.

\section{Plasmid construction and parasite transfection}

We modified the endogenous TgPRMT5 genomic locus to contain additional sequence at the $3^{\prime}$ end that added a $3 \mathrm{X}$ hemagglutinin (HA) epitope onto the C-terminus of TgPRMT5. Pru $\Delta k u 80$ genomic DNA was used to amplify a $1.5 \mathrm{~kb}$ fragment at the PRMT5 $3^{\prime}$ end using the primers PRMT5HA_F (5'-TAC TTC CAA TCC AAT TTA ATG CGT CTC TAC CAC TGC GTT TTC C-3') and PRMT5HA_R (5'-TCC TCC ACT TCC AAT TTT AGC TTT TCC GAT GAA GTAA TGT TT-3') containing ligation-independent cloning sequences (underlined). This PRMT5 fragment was inserted into the pLIC_ HAx3_DHFRTs endogenous tagging vector such that the TgPRMT5 coding sequence was fused in frame with the epitope coding region. The pLIC_PRMT5_HAx3 DHFRTs construct was confirmed by sequencing. For transfection, $30 \mu \mathrm{g}$ pLIC_PRMT5_HAx3_DHFRTs plasmid was linearized by overnight digestion with SgrAI within the PRMT5 homologous region followed by ethanol precipitation. Pru $\Delta k u 80$ tachyzoites were transformed with the linearized construct by electroporation. Subsequent to overnight growth in the HFF, parasite cultures were treated with $1.0 \mu \mathrm{M}$ pyrimethamine [13]. Drug-resistant parasites were cloned by limiting dilution and screened using western blot and immunofluorescence for expression of HA-tagged TgPRMT5.

\section{Protein expression and purification}

Full-length cDNAs encoding TgH3 (TGGT1_261240) and TgH4 (TGGT1_239260) were amplified by polymerase chain reaction using the primer pairs TgH3_F/ TgH3_R (5'-AAA GAA TTC ATG GCG CGC ACC AAG $\left.-3^{\prime}\right) /\left(5^{\prime}\right.$-TCC GAG CTC TTA AGA CCG TTC ACC AC-3') and TgH4_F/TgH4_R (5'-AAA GAA TTC ATG TCG GGC CGA GGC AAG-3')/(5'-TCC GAG CTC TTA ACC ACC GAA ACC GTA G-3') and cloned at the 
EcoRI and SacI sites of vector PET-32a (Novagen, Madison, WI, USA) according to the manufacturer's instructions to produce His-TgH3 and His-TgH4. Recombinant proteins were expressed in E.coli strain BL21 (DE3). Induction was performed by adding $0.1 \mathrm{mM}$ IPTG for 4 $\mathrm{h}$ at $37{ }^{\circ} \mathrm{C}$. Recombinant proteins were purified from $1 \mathrm{l}$ of culture using Ni-NTA Fast Start Kit (Sigma-Aldrich, Taufkirchen, Germany). Purified recombinant $\mathrm{TgH} 3$ and $\mathrm{TgH} 4(\mathrm{rTgH} 3$ and $\mathrm{rTgH} 4)$ proteins were used for the in vitro methylation assay.

\section{Western blot}

Tachyzoites were purified from infected HFF monolayers by scraping and passage through an $18 \mathrm{G}$ needle 10 times. Bradyzoites were purified from host cell debris by filtration through a $25 \mathrm{~mm}$ Nuclepore Track-Etched Polycarbonate Membrane circle with a $3.0 \mu \mathrm{m}$ pore size (GE Healthcare, Madison, WI, USA) into a conical tube. Parasites were then pelleted by centrifugation and washed with cold PBS at $4{ }^{\circ} \mathrm{C}$. Antibody to Toxoplasma BAG1 (1:1000) was used to detect protein-specific expression in the bradyzoites. Toxoplasma $\beta$-tubulin expression detected by specific polyclonal antibody (1:1000) served as a protein loading control. The antibody to BAG1 and anti- $\beta$-tubulin antibody were provided by Dr David Sibley (Washington University, St. Louis, MO, USA) and Dr Louis Weiss (Albert Einstein College of Medicine, NY, USA) respectively. To estimate the distribution of TgPRMT5 in the cytoplasmic and nuclear compartments of parasites, subcellular fractionations were performed using the NE-PER ${ }^{\mathrm{TM}}$ nuclear and cytoplasmic extraction kit (Pierce, Rockford, IL, USA) according to the manufacturer's instructions. Parasite lysates $(20 \mu \mathrm{g})$ of tachyzoites or bradyzoites were separated by SDS/PAGE $(10 \%)$ and transferred to nitrocellulose membranes. Western blots were carried out using rat anti-HA antibody (Roche, CA, USA) (1:2000) and rabbit anti-H4 antibody (Merck Millipore, Darmstadt, Germany) (1:1000) as primary antibodies and horseradish peroxidase-conjugated goat anti-rat IgG (1:3000) and anti-rabbit IgG (1:3000) as secondary antibodies. The results were visualized using the ECL detection system (GE Healthcare).

\section{Indirect immunofluorescent assay (IFA)}

For IFA, the infected HFF monolayers grown on coverslips were fixed in $4 \%$ paraformaldehyde for $20 \mathrm{~min}$ at room temperature. They were then permeabilized for 10 min in PBS containing 3\% BSA and 0.2\% Triton $\mathrm{X}-100$ and blocked for $1 \mathrm{~h}$ in PBS with 3\% BSA. The infected HFF monolayers were first probed with rabbit anti-HA antibody (Sigma) (1:500) and anti-BAG1 antibody (1:100). Secondary antibodies were FITC-labeled anti-rabbit IgG (Sigma-Aldrich, Taufkirchen, Germany) and TRITC-labeled anti-mouse IgG (Sigma-Aldrich, Taufkirchen, Germany). Fluorescent images were obtained using a Nikon ECLIPSE E600 epifluorescence microscope.

\section{Immunoprecipitation}

To isolate epitope-tagged proteins, immunoprecipitations were performed using parasite lysates. Typically, 300-500 $\mu$ g lysate was used for each immunoprecipitation reaction. Rat anti-HA antibody (Roche) was used for coupling with the Pierce Crosslink IP Kit (Thermo Fisher Scientific, Rockford, IL, USA) according to the manufacturer's instructions. Lysates of the TgPRMT5-HA strain were immunoprecipitated with anti-HA antibody. The immunoprecipitated complexes were then used for an in vitro methylation assay.

\section{In vitro methylation assay}

In vitro methylation assays were performed at $30{ }^{\circ} \mathrm{C}$ for $2 \mathrm{~h}$ in $20 \mu \mathrm{l}$ methylation-assay buffer $(50 \mathrm{mM}$ Tris- $\mathrm{HCl}$, $\mathrm{pH} 8.5,20 \mathrm{mM} \mathrm{KCl}, 10 \mathrm{mM} \mathrm{MgCl} 2,1 \mathrm{mM} \mathrm{DTT}, 250 \mathrm{mM}$ sucrose and $1 \mathrm{mM}$ PMSF) containing $5 \mu \mathrm{g}$ free calf core histones ( $\mathrm{H} 2 \mathrm{~A}, \mathrm{H} 2 \mathrm{~B}, \mathrm{H} 3$ and $\mathrm{H} 4)$ or $2 \mu \mathrm{g}$ purified thioredoxin $-6 \times$ His $T$. gondii core histones $\mathrm{H} 3(\mathrm{rTgH} 3)$ and $\mathrm{H} 4(\mathrm{rTgH} 4)$ as substrates and $2 \mu \mathrm{g}$ purified HA-tagged TgPRMT5. Reactions were stopped by the addition of LDS sample buffer $(4 \times)$ and boiling for 5 min. Proteins were resolved by SDS/PAGE (15\% gel) and transferred onto PVDF membranes. Immunoblot assays were performed using the following rabbit polyclonal antibodies: anti-monomethyl H3R26 (ab130898; Abcam, CA, USA), anti-symmetric dimethyl H3R26 (ab127095; Abcam, CA, USA), anti-monomethyl H4R3 (ab17339; Abcam, CA, USA), and anti-symmetric dimethyl H4R3 (ab5823; Abcam, CA, USA) at $1 \mu \mathrm{g} / \mathrm{ml}$ concentration and horseradish peroxidase-conjugated goat anti-rabbit IgG (Boster Bio-Tech, Wuhan, China) (1:2000) as the secondary antibody. The results were visualized with the ECL detection system.

\section{Results}

\section{Fusion of HA-tag to endogenous TgPRMT5}

To study the expression and subcellular localization of TgPRMT5 in tachyzoites and bradyzoites, we tagged the $3^{\prime}$ end of the endogenous genomic locus with sequence encoding a 3xHA tag in Pru $\triangle k u 80$ parasites (Fig. 1a). Successful tagging of the endogenous TgPRMT5 was confirmed by IFA and western blot. Western blot using anti-HA antibody detected a specific protein band of $110 \mathrm{kDa}$, consistent with the predicted size of TgPRMT5 (Fig. 1b). By contrast, this protein band was not detected in the control wild-type Pru strain parasites. Western blot revealed that TgPRMT5 was expressed in both 

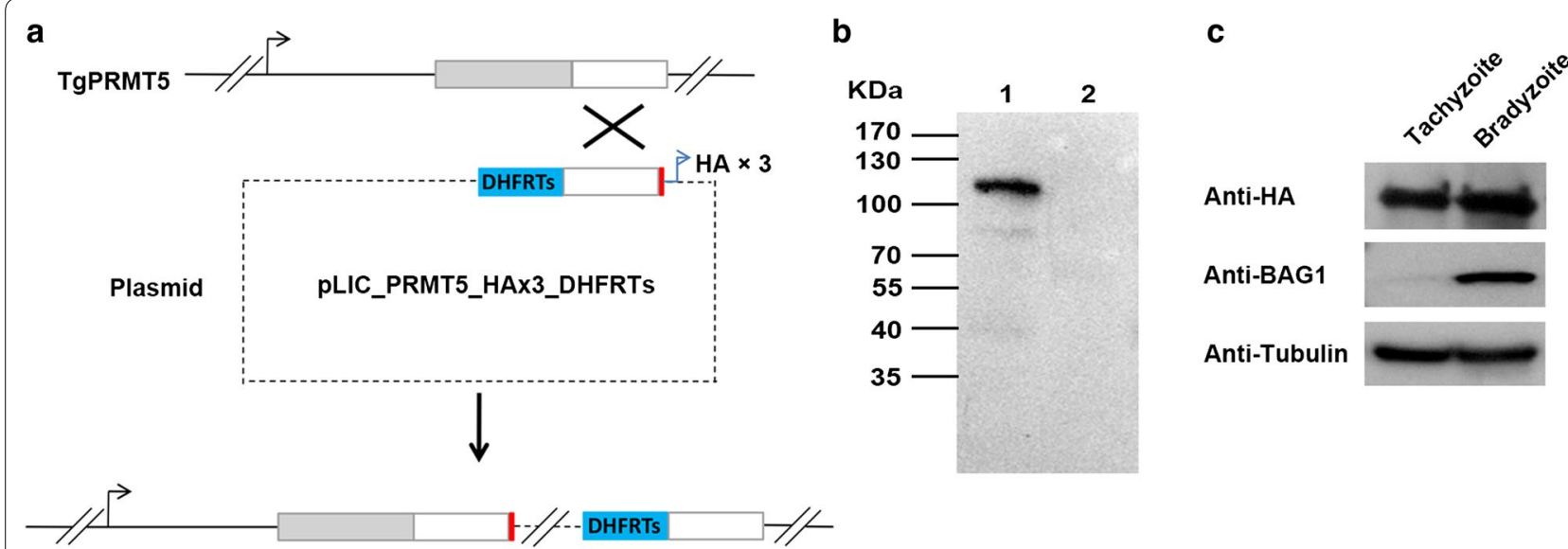

Fig. 1 Confirmation of the C-terminal HA-tagging of the endogenous TgPRMT5 locus in the Pru strain. a Predicted integration event of HA fusion at the endogenous TgPRMT5 locus. Top: TgPRMT5 locus on chromosome X. Grey boxes represent the front part of TgPRMT5 gene; white boxes represent the TgPRMT5 region used for homologous recombination in the transfection plasmid; red boxes indicate HAx3-tagged. Middle: the plasmid pLIC_PRMT5_HAx3_DHFRTs. Bottom: The resultant single-crossover event at the TgPRMT5 locus with integration of one copy of the plasmid. $\mathbf{b}$ Western blot with anti-HA antibody that bind to the HA-tag. The band of $110 \mathrm{kDa}$ was detected in the HA-tagged TgPRMT5 clone. Lane 1: clone of HA-tagged TgPRMT5; Lane 2: wild-type Pru strain as negative control. c Expression of TgPRMT5 in tachyzoites and bradyzoites. The two stages of the parasite were differentiated by antibody against BAG1, a protein expressed specifically in the bradyzoite stage. Anti- $\beta$-tubulin antibody served as a protein loading control

tachyzoites and bradyzoites with slight enrichment in the latter stage (Fig. 1c).

\section{Subcellular localization of TgPRMT5}

IFA was performed to check subcellular localization of TgPRMT5, and the TgBAG1 was specifically expressed in the cytoplasm in the bradyzoite stage as a bradyzoite marker. IFA results showed that TgPRMT5 was localized in the cytoplasm in the tachyzoite stage; however, it was mainly localized in the nucleus in the bradyzoite stage (Fig. 2a). Further analysis of the nuclear-cytoplasmic distribution of TgPRMT5 was performed using fractionated parasite lysates (Fig. 2b). Parasites were separated into nuclear and cytoplasmic fractions, and proteins from each fraction were resolved by SDS/PAGE and subjected to immunoblotting. The fidelity of the fractionation was confirmed by the predominant detection of histone $\mathrm{H} 4$ in the nuclear fraction (Fig. 2b). Consistent with the IFA observations, TgPRMT5 shifted from cytosolic to nuclear localization during conversion to the bradyzoite stage.
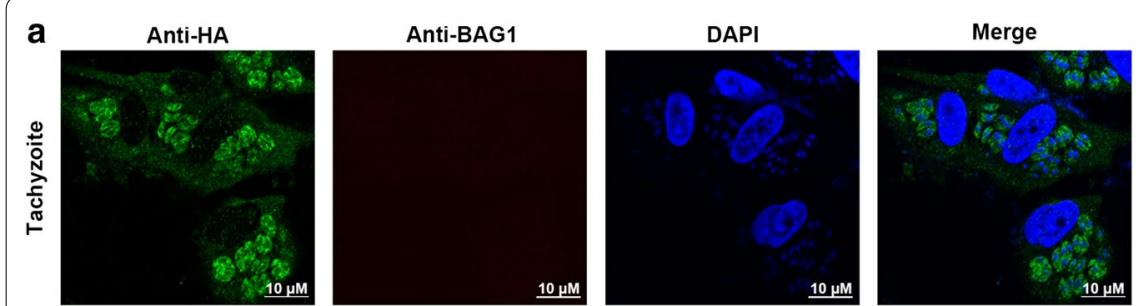

b
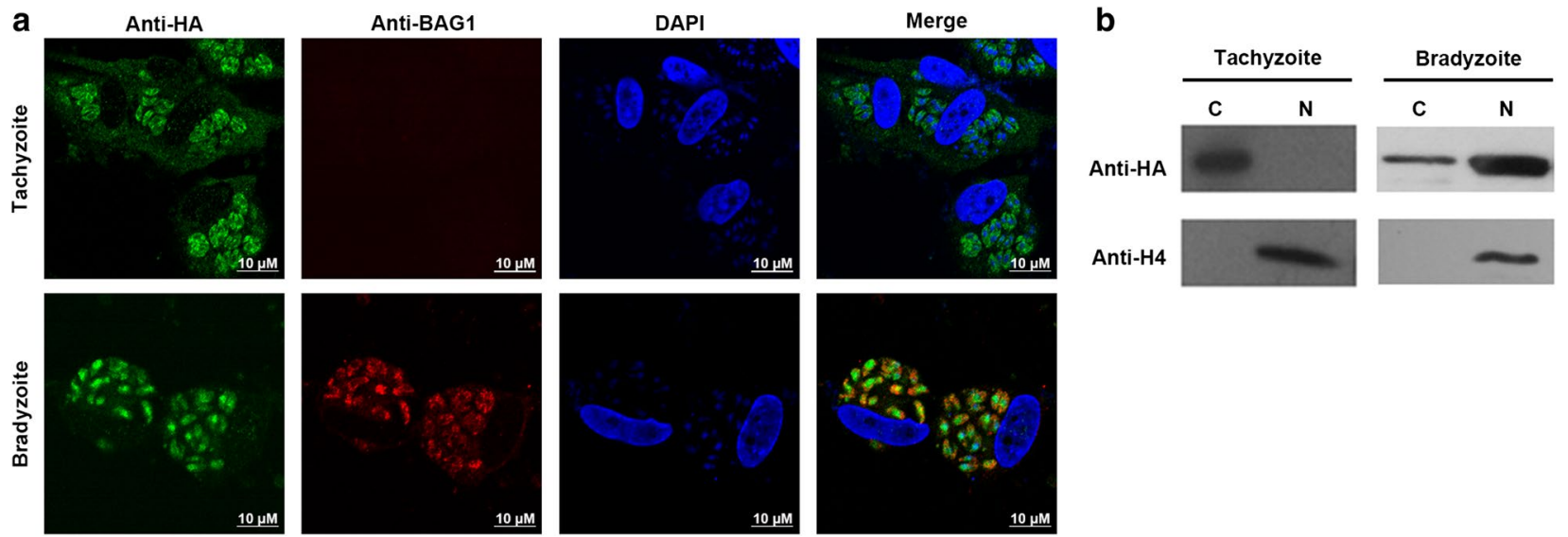

Fig. 2 Subcellular localization of TgPRMT5 in tachyzoites and bradyzoites. a Localization of HA-TgPRMT5. Representative images of HA-TgPRMT5 in tachyzoites and bradyzoites. Nuclei were stained with DAPI. b Western blots of the parasite nuclear (N) and cytoplasmic (C) fractions, separated by 10\% SDS-PAGE, and probed with anti-HA antibody (upper panel), and anti-H4 antibody (lower panel). Scale-bars: $10 \mu \mathrm{m}$ 


\section{TgPRMT5 enzyme activity}

To determine whether endogenous TgPRMT5 has arginine methyltransferase activity, calf core histones H2A, $\mathrm{H} 2 \mathrm{~B}, \mathrm{H} 3$ and $\mathrm{H} 4$ as well as purified T. gondii recombinant histones $\mathrm{H} 3$ and $\mathrm{H} 4$ (rTgH3 and $\mathrm{rTgH} 4)$ were used as substrates in the methylation assay. Endogenous TgPRMT5-HA was purified using protein A/G beads with HA antibody. The purified products were checked in the SDS/PAGE gel and stained by Commassie Blue Staining Solution. The size of the main band was around 110 $\mathrm{kDa}$, consistent with the predicted size of TgPRMT5-HA (Fig. 3a). Specific antibodies were used against monomethylated (MMA) and symmetrically dimethylated (SDMA) H3R26 or H4R3 to monitor TgPRMT5 activity by immunoblotting. Endogenous TgPRMT5-HA was shown to catalyze both H3R26 and H4R3 monomethylation and symmetric methylation, consistent with the prediction of this protein as a PRMT5 homolog (Fig. 3b).

\section{Discussion}

Arginine methylation governs important cellular processes that impact growth, proliferation, differentiation, and development [9]. PRMT5 is the major type II methyltransferase and is widely distributed and highly conserved [14]. Unique among the PRMTs, PRMT5 works in concert with other cellular proteins including ATPdependent chromatin remodelers and co-repressors to induce epigenetic silencing. Furthermore, PRMT5 has recently been implicated in control of growth-promoting and pro-survival pathways, thereby demonstrating its versatile enzymatic roles in epigenetic regulation of anticancer target genes and organelle biogenesis [9].
In the present study, we presented biochemical evidence demonstrating that TgPRMT5 has intrinsic type II PRMT activity, catalyzing the formation of MMA and SDMA. In the histone $\mathrm{H} 4 \mathrm{~N}$-terminus, TgPRMT5 can symmetrically methylate $\mathrm{Arg}^{3}$, a transcription repression marker [15]. These findings suggest that TgPRMT5 protein is a PRMT5 homolog and may participate in gene repression in T. gondii. TgPRMT5 was expressed in the tachyzoite and bradyzoite stages of the parasite, with the protein localization in cytoplasm in tachyzoites; however, it mainly localized in the nucleus in bradyzoites. In mammals, localization of PRMT5 differs between nontransformed and transformed cells. In most primary and immortalized cells, PRMT5 is primarily located in the cytosol with small amounts in the nucleus. In contrast, this distribution is reversed in transformed cells [16-18]. Therefore, PRMT5 localization and the substrates it targets in each cellular compartment appear to play a crucial role in the control of cell growth and proliferation. The differential subcellular localization of TgPRMT5 in tachyzoites and bradyzoites suggests that it may play a role in tachyzoite-bradyzoite conversion.

\section{Conclusions}

In present study, we determined the expression and cellular localization of TgPRMT5 in tachyzoites and bradyzoites and confirmed its type II PRMT activity. We demonstrated the major changes in expression and cellular localization of TgPRMT5 during the tachyzoite and bradyzoite stages in T. gondii. These findings will provide a novel insight into addressing the mechanism of tachyzoite-bradyzoite transformation.

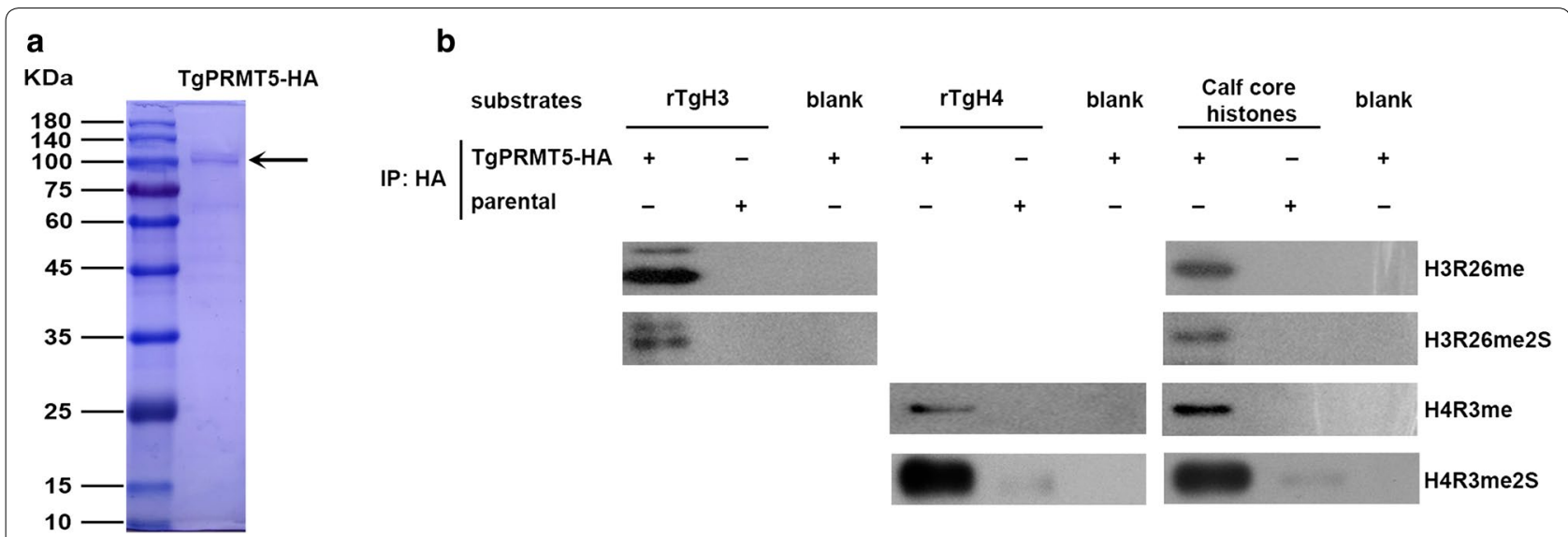

Fig. 3 In vitro methylation of histones by purified HA-tagged endogenous TgPRMT5. a The HA-tagged endogenous TgPRMT5 were purified with an anti-HA antibody and checked in SDS/PAGE. $\mathbf{b}$ Immunoprecipitations using an anti-HA antibody were performed on parasite lysates made from TgPRMT5-HA and the parental Pru $\triangle$ ku80 line. The immunoprecipitated products were used in vitro methylation assays with T. gondii recombinant histones $\mathrm{H3} / \mathrm{H} 4$ ( $\mathrm{rTgH} 3$ and $\mathrm{rTgH} 4$ ) and calf core histones as substrates or without substrates (blank), and the reactions were separated by SDS/PAGE (15\% gel). Immunoblots were performed using rabbit polyclonal antibodies as follows: anti-monomethyl H3R26, anti-symmetric dimethyl H3R26, anti-monomethyl H4R3 and anti-symmetric dimethyl H4R3 


\section{Abbreviations}

PRU: Prugniaud; IFA: indirect immunofluorescent assay; PRMT: protein arginine methyltransferase; MMA: monomethylated; SDMA: symmetrically dimethylated.

\section{Acknowledgements}

We are grateful to Dr. David Sibley (Washington University, St. Louis, MO) for providing the anti- $\beta$-tubulin antibody and Dr. Louis Weiss (Albert Einstein College of Medicine, NY) for the antibody to BAG1 and Pru $\triangle k u 80$ parasite strain.

\section{Funding}

This study is supported by National Nature Science Foundation of China (No. 81601779 and No. 31030066), Guangdong Natural Science Foundation (No. 2014A030310210), Science and Medical Science and Technology Research Project of Guangdong Province (No. A2016029), Science and Technology Planning Project of Guangdong Province (No. 2013B060300031). Research in the Sullivan laboratory is supported by a National Institutes of Health Grant (Al1 16496).

\section{Availability of data and materials}

Data supporting the conclusions of this article are included within the article.

\section{Authors' contributions}

$X-G C$ and $M L$ conceived of the study. M L wrote the manuscript. ML supervised the entire work and performed the plasmid construction and parasite transfection. F-X L carried out the cell cultures and took the methylation assay and indirect immunofluorescent assay. $M L$ and $F-X L$ prepared all of the figures. C-Y L, X-C L, L-F C, KW, P-LY and Z-F L performed the protein expression and purification. T-K L provided technical support. WJS and L-W C critically revised the manuscript. All authors read and approved the final manuscript..

Ethics approval and consent to participate

Not applicable.

\section{Consent for publication}

Not applicable.

\section{Competing interests}

The authors declare that they have no competing interests.

\section{Publisher's Note}

Springer Nature remains neutral with regard to jurisdictional claims in published maps and institutional affiliations

\section{Author details}

${ }^{1}$ Department of Pathogen Biology, Guangdong Provincial Key Laboratory of Tropical Disease Research, School of Public Health, Southern Medical University, Guangzhou 510515, Guangdong, People's Republic of China. ${ }^{2}$ Laboratory Animal Research Center, Nanfang Hospital, Southern Medical University, Guangzhou 510515, Guangdong, People's Republic of China. ${ }^{3}$ Futian Center for disease control and prevention, Shenzhen 518040, Guangdong, People's Republic of China. ${ }^{4}$ Department of Pharmacology \& Toxicology, Indiana University School of Medicine, Indianapolis, IN, USA. ${ }^{5}$ Department of Microbiology \& Immunology, Indiana University School of Medicine, Indianapolis, IN, USA. ${ }^{6}$ Department of Entomology, Pennsylvania State University, 501 ASI Building, University Park, PA 16802, USA.
Received: 16 January 2019 Accepted: 29 April 2019

Published online: 08 May 2019

\section{References}

1. Hill D, Dubey JP. Toxoplasma gondii: transmission, diagnosis and prevention. Clin Microbiol Infect. 2002;8:634-40.

2. Dixon SE, Stilger KL, Elias EV, Naguleswaran A, Sullivan WJ Jr. A decade of epigenetic research in Toxoplasma gondii. Mol Biochem Parasitol. 2010;173:1-9.

3. Nardelli SC, Che FY, de Monerri NC, Xiao H, Nieves E, Madrid-Aliste C, et al. The histone code of Toxoplasma gondii comprises conserved and unique posttranslational modifications. MBio. 2013;4:e00922.

4. El Bissati K, Suvorova ES, Xiao H, Lucas O, Upadhya R, Ma Y, et al. Toxoplasma gondii arginine methyltransferase 1 (PRMT1) is necessary for centrosome dynamics during tachyzoite cell division. MBio. 2016;7:e02094.

5. Saksouk N, Bhatti MM, Kieffer S, Smith AT, Musset K, Garin J, et al. Histone-modifying complexes regulate gene expression pertinent to the differentiation of the protozoan parasite Toxoplasma gondii. Mol Cell Biol. 2005;25:10301-14.

6. Musiyenko A, Majumdar T, Andrews J, Adams B, Barik S. PRMT1 methylates the single Argonaute of Toxoplasma gondii and is important for the recruitment of Tudor nuclease for target RNA cleavage by antisense guide RNA. Cell Microbiol. 2012;14:882-901.

7. Schurter BT, Koh SS, Chen D, Bunick GJ, Harp JM, Hanson BL, et al. Methylation of histone $\mathrm{H} 3$ by coactivator-associated arginine methyltransferase 1. Biochemistry. 2001;40:5747-56.

8. Cosgrove MS, Boeke JD, Wolberger C. Regulated nucleosome mobility and the histone code. Nat Struct Mol Biol. 2004;11:1037-43.

9. Karkhanis V, Hu YJ, Baiocchi RA, Imbalzano AN, Sif S. Versatility of PRMT5induced methylation in growth control and development. Trends Biochem Sci. 2011:36:633-41.

10. Bedford MT. Arginine methylation at a glance. J Cell Sci. 2007;120:4243-6.

11. Durcova-Hills G, Tang F, Doody G, Tooze R, Surani MA. Reprogramming primordial germ cells into pluripotent stem cells. PLoS ONE. 2008;3:e3531.

12. Eguizabal C, Shovlin TC, Durcova-Hills G, Surani A, McLaren A. Generation of primordial germ cells from pluripotent stem cells. Differentiation. 2009:78:116-23.

13. Zhang G, Huang X, Boldbaatar D, Battur B, Battsetseg B, Zhang H, et al. Construction of Neospora caninum stably expressing TgSAG1 and evaluation of its protective effects against Toxoplasma gondii infection in mice. Vaccine. 2010;28:7243-7.

14. Wang YC, Li C. Evolutionarily conserved protein arginine methyltransferases in non-mammalian animal systems. FEBS J. 2012;279:932-45.

15. Wysocka J, Allis CD, Coonrod S. Histone arginine methylation and its dynamic regulation. Front Biosci. 2006;11:344-55.

16. Pal S, Vishwanath SN, Erdjument-Bromage $H$, Tempst P, Sif S. Human SWI/ SNF-associated PRMT5 methylates histone $\mathrm{H} 3$ arginine 8 and negatively regulates expression of ST7 and NM23 tumor suppressor genes. Mol Cell Biol. 2004:24:9630-45.

17. Wang L, Pal S, Sif S. Protein arginine methyltransferase 5 suppresses the transcription of the RB family of tumor suppressors in leukemia and lymphoma cells. Mol Cell Biol. 2008;28:6262-77.

18. Pal S, Baiocchi RA, Byrd JC, Grever MR, Jacob ST, Sif S. Low levels of miR92b/96 induce PRMT5 translation and H3R8/H4R3 methylation in mantle cell lymphoma. EMBO J. 2007:26:3558-69. 